\title{
Electrophilic trifluoromethylselenolation of terminal alkynes with Se-(trifluoromethyl) 4-methylbenzenesulfonoselenoate
}

\author{
Clément Ghiazza ${ }^{1}$, Anis Tlili ${ }^{* 1}$ and Thierry Billard ${ }^{* 1,2}$
}

\author{
Full Research Paper \\ Address: \\ ${ }^{1}$ Institute of Chemistry and Biochemistry, Univ Lyon, Université Lyon \\ 1, CNRS, 43 Bd du 11 novembre 1918, F-69622 Villeurbanne, France \\ and ${ }^{2}$ CERMEP-In vivo Imaging, Groupement Hospitalier Est, 59 Bd \\ Pinel, F-69003 Lyon, France \\ Email: \\ Anis Tlili* - anis.tlili@univ-lyon1.fr; Thierry Billard* - \\ Thierry.billard@univ-lyon1.fr \\ ${ }^{*}$ Corresponding author \\ Keywords: \\ alkynes; nucleophilic addition; perfluoroalkylselenolation; \\ Se-(trifluoromethyl) 4-methylbenzenesulfonoselenoate; \\ trifluoromethylselenolation
}

Open Access

\author{
Beilstein J. Org. Chem. 2017, 13, 2626-2630. \\ doi: $10.3762 /$ bjoc. 13.260 \\ Received: 22 September 2017 \\ Accepted: 21 November 2017 \\ Published: 07 December 2017 \\ This article is part of the Thematic Series "Organo-fluorine chemistry IV". \\ Guest Editor: D. O'Hagan
}

(C) 2017 Ghiazza et al.; licensee Beilstein-Institut.

License and terms: see end of document.

\begin{abstract}
Herein the nucleophilic addition of Se-(trifluoromethyl) 4-methylbenzenesulfonoselenoate, a stable and easy-to-handle reagent, to alkynes is described. This reaction provides trifluoromethylselenylated vinyl sulfones with good results and the method was extended also to higher fluorinated homologs. The obtained compounds are valuable building blocks for further syntheses of fluoroalkylselenolated molecules.
\end{abstract}

\section{Introduction}

Over the last decades, fluorinated compounds have been the subject of growing interest $[1,2]$. The specific properties introduced by fluorinated groups have contributed to the "success story" of fluorinated molecules. Nowadays, fluorinated compounds find applications in various fields, from life sciences to materials [3-15]. In the objective to design new molecules with specific properties, novel fluorinated substituents have been developed, such as diverse trifluoromethylchalcogeno groups, due to their particular electronic properties [16] and, more especially, to their high lipophilicity [17]. Whereas the $\mathrm{CF}_{3} \mathrm{O}$ and $\mathrm{CF}_{3} \mathrm{~S}$ substituents have been largely studied [18-23], the $\mathrm{CF}_{3} \mathrm{Se}$ group, albeit known, has gained only little attention until recently. However, selenylated derivatives present pertinent properties and have found some interest in materials [24], life sciences [25-33] and drug design [34-37]. Furthermore, very recently, the Hansch lipophilicity parameter of $\mathrm{CF}_{3} \mathrm{Se}$ has been determined $\left(\pi_{R}=1.29\right)$ - a high value lying between that of $\mathrm{CF}_{3} \mathrm{O}$ and $\mathrm{CF}_{3} \mathrm{~S}$ [38]. Consequently, trifluoromethylselenolated molecules could represent interesting alternatives in the modulation of properties for various applications.

Despite such potential interest for $\mathrm{CF}_{3} \mathrm{Se}$ compounds, methods to their syntheses remain still limited [39]. Direct trifluoromethylselenolation reactions have recently gained renewed 
interest and mainly follow two strategies. The nucleophilic approach is based on the use of the $\mathrm{CF}_{3} \mathrm{Se}^{-}$anion which must be prepared from stoichiometric amounts of metallic selenium [40-53]. Concerning the electrophilic approach, two reagents, that are easy to obtain, have been described: $\mathrm{CF}_{3} \mathrm{SeCl}$ [38,54-67] and $\mathrm{CF}_{3} \mathrm{SeTs}$ [68].

\section{Results and Discussion}

Recently, we have described the electrophilic addition of $\mathrm{CF}_{3} \mathrm{SeCl}$ to alkenes to access $\alpha$-chloro- $\beta$-trifluoromethylselenolated molecules [65]. These products are particularly interesting because the presence of the chlorine substituent opens the way to post-functionalization and thereby to syntheses of more elaborated compounds. However, the similar reaction with alkynes has failed and only a complex mixture was observed which is basically due to the high reactivity of $\mathrm{CF}_{3} \mathrm{SeCl}$.

To overcome this issue, we have developed another easier-touse reagent with a more controlled reactivity to perform electrophilic trifluoromethylselenolations, namely Se-(trifluoromethyl) 4-methylbenzenesulfonoselenoate (1a). This reagent is easily obtained by reacting sodium toluenesulfinate with in situ formed $\mathrm{CF}_{3} \mathrm{SeCl}$ [68]. With this reagent at hand we envisaged the trifluoromethylselenolation of alkynes.

To our delight, the addition of $\mathbf{1 a}$ to phenylacetylene (2a) at room temperature, without any other practical precautions, lead to the expected addition product $\mathbf{3 a}$ with good yield (Scheme 1).

Subsequently, the reaction was extended to various arylalkynes and afforded, in general, good yields. Satisfactory to excellent results were observed whatever the electronic character (donor or acceptor) of the substituents on the phenyl moiety were. Even highly electron-withdrawing groups led to satisfactory yields of the products $\mathbf{3 h}-\mathbf{j}$. The reaction is also not too sensitive to steric hindrance because good results were obtained also with ortho-substituted substrates 3c,e,i. Nevertheless, the reaction with aliphatic alkyne $\mathbf{2 k}$, resulted in a low yield $(28 \%)$.

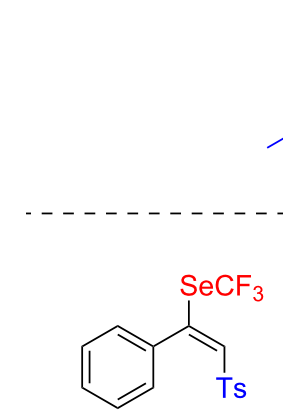

$3 \mathbf{a}$

$74 \%(88 \%)$<smiles>FC(F)(F)[Ge]C(=C[As])c1ccccc1Cl</smiles>

3 e

$85 \%(79 \%)$<smiles>Cc1ccc(S(=O)(=O)[Se]C(F)(F)F)cc1</smiles>

1 a (1.1 equiv)

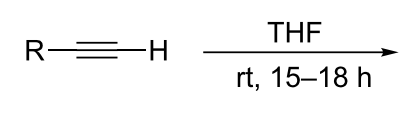

2<smiles>[R]/C([SeH])=C\[13CH3]</smiles>

3<smiles>COc1ccc(/C(=C\[13F])[Se]C(F)(F)F)cc1</smiles>

3b $85 \%(83 \%)$<smiles>COc1ccccc1/C(=C\[As])C(F)(F)F</smiles>

3c $85 \%(95 \%)$<smiles>[3H]/C=C(/[Se]C(F)(F)F)c1ccc(CC#N)cc1</smiles>

3d<smiles>Fc1cc(/C(=C\[123I])C(F)(F)F)cc(F)c1F</smiles>

$81 \%(88 \%)$<smiles>FC(F)(F)[Ge]C(=C[As])c1ccc(Br)cc1</smiles>
$3 \mathbf{f}$ $51 \%(59 \%)$<smiles>[3H]C=C(c1ccc(C(C)=O)cc1)C(F)(F)F</smiles>

$3 \mathrm{~g}$ $65 \%(81 \%)$<smiles>[3H]C=C(c1cc(C(F)(F)F)cc(C(F)(F)F)c1)C(F)(F)F</smiles>

3h<smiles>[3H]C=C(c1ccccc1OC(F)(F)F)C(F)(F)F</smiles>

$3 \mathbf{i}$ $53 \%(68 \%)$

Scheme 1: Electrophilic addition of 1a to alkynes. Yields shown are those of isolated products; yields determined by ${ }^{19} \mathrm{~F} \mathrm{NMR}$ spectroscopy with $\mathrm{PhOCF}_{3}$ as an internal standard are shown in parentheses. 
The reaction is stereoselective with the exclusive formation of the trans-isomers. Further, a high regioselectivity is observed but, surprisingly, the anti-Markovnikov regioisomers were obtained. The stereochemistry and regiochemistry were confirmed thanks to the X-ray structure of compound 3a (Figure 1).

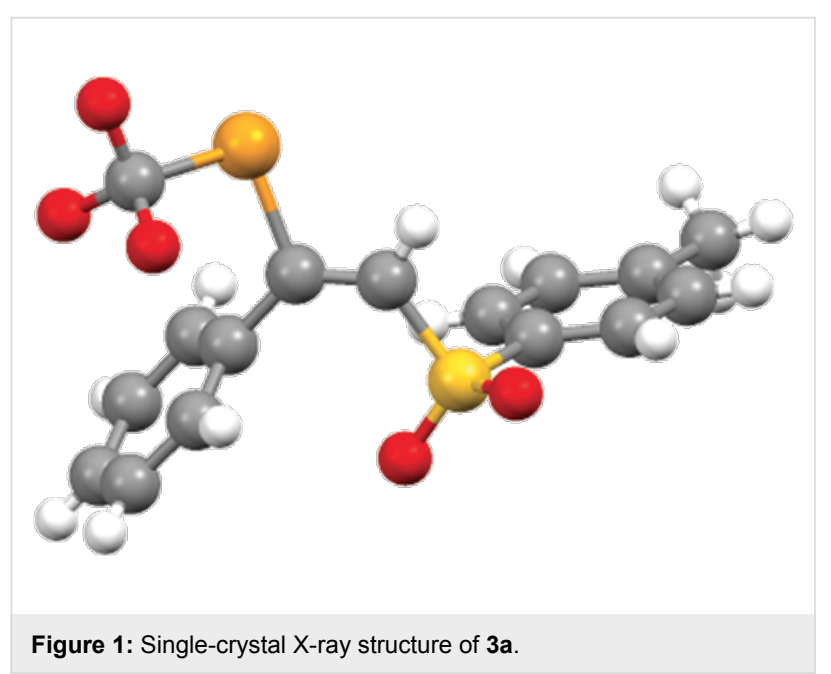

From a mechanistic point of view, the reaction starts certainly with the intermediate formation of the trifluoromethylselenonium ion $\mathbf{I}$. This is in accordance with the observed trans-selectivity due to the anti opening of $\mathbf{I}$ by the toluenesulfinate anion. The regioselectivity could be rationalized by the steric hindrance between the aromatic moiety and the large nucleophile (toluenesulfinate anion) which favors the attack on the less hindered side (Scheme 2, pathway a). Such sensitivity to steric hindrance of I was confirmed by the lack of reactions observed with internal alkynes.

Higher homologs of reagent 1a are also available, the reaction was briefly performed with $\mathbf{1 b}$ and $\mathbf{1 c}$ (Scheme 3). The expected products were obtained with good yields. The tridecafluorohexylselenyl group in product 5a makes this compound interesting because it opens the way to applications in the design of fluorinated polymers or surfactants.

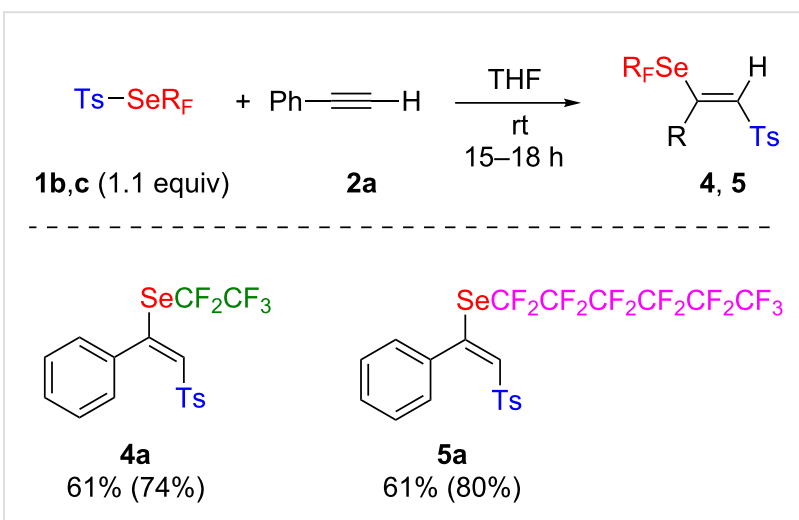

Scheme 3: Perfluoroalkylselenolation of alkynes. Yields shown are those of isolated products; yields determined by ${ }^{19} \mathrm{~F}$ NMR spectroscopy with $\mathrm{PhOCF}_{3}$ as an internal standard are shown in parentheses.

\section{Conclusion}

To conclude, Se-(trifluoromethyl) 4-methylbenzenesulfonoselenoate and $S e$-(perfluoroalkyl) 4-methylbenzenesulfonoselenoate have been confirmed as valuable bench-stable reagents to perform perfluoroalkylselenolations. Nucleophilic additions with alkynes to provide perfluoroalkylselenolated vinyl sulfones can easily be carried out. Se-(Trifluoromethyl) 4-methylbenzenesulfonoselenoate and $\mathrm{Se}$-(perfluoroalkyl) 4-methylbenzenesulfonoselenoate constitute interesting building blocks for various applications.

\section{Experimental}

Typical procedure: To a flask equipped with a magnetic stir bar were added 1 ( $0.25 \mathrm{mmol}, 1.1$ equiv), alkyne 2 ( $0.23 \mathrm{mmol}$, 1.0 equiv), and anhydrous THF $(1 \mathrm{~mL})$. The reaction was stirred at $25{ }^{\circ} \mathrm{C}$ for $15-18$ hours (conversion was checked by ${ }^{19} \mathrm{~F}$ NMR with $\mathrm{PhOCF}_{3}$ as internal standard). The crude residue was purified by chromatography to afford the desired products $\mathbf{3}-\mathbf{5}$.<smiles>Cc1ccc(S(=O)(=O)[Se][Se](=O)(=O)c2ccc(C)cc2)cc1</smiles> 


\section{Supporting Information}

\section{Supporting Information File 1}

Additional experimental and analytical data and NMR spectra.

[http://www.beilstein-journals.org/bjoc/content/ supplementary/1860-5397-13-260-S1.pdf]

\section{Acknowledgements}

Clément Ghiazza holds a doctoral fellowship from la Région Rhône Alpes. The authors are grateful to the CNRS and the French Ministry of Research for financial support. The French Fluorine Network is also acknowledged for its support. We thank Dr. Erwann Jeanneau (Centre de Diffractométrie Henri Longchambon) for collecting the crystallographic data and solving the structure of $\mathbf{3 a}$.

\section{ORCID ${ }^{\circledR}$ iDs}

Clément Ghiazza - https://orcid.org/0000-0002-1264-2559

Anis Tlili - https://orcid.org/0000-0002-3058-2043

Thierry Billard - https://orcid.org/0000-0002-2937-9523

\section{References}

1. Dolbier, W. R., Jr. J. Fluorine Chem. 2005, 126, 157-163. doi:10.1016/j.jfluchem.2004.09.033

2. Kirsch, P. Introduction. Modern Fluoroorganic Chemistry, 2nd ed.; Wiley-VCH: Weinheim, Germany, 2013; pp 1-24.

3. Becker, A. Inventory of Industrial Fluoro-biochemicals; Eyrolles: Paris, 1996.

4. Smart, B. E. J. Fluorine Chem. 2001, 109, 3-11. doi:10.1016/S0022-1139(01)00375-X

5. Hagmann, W. K. J. Med. Chem. 2008, 51, 4359-4369 doi:10.1021/jm800219f

6. Wang, J.; Sánchez-Roselló, M.; Aceña, J. L.; del Pozo, C.; Sorochinsky, A. E.; Fustero, S.; Soloshonok, V. A.; Liu, H. Chem. Rev. 2014, 114, 2432-2506. doi:10.1021/cr4002879

7. Gillis, E. P.; Eastman, K. J.; Hill, M. D.; Donnelly, D. J.; Meanwell, N. A. J. Med. Chem. 2015, 58, 8315-8359. doi:10.1021/acs.jmedchem.5b00258

8. Zhou, Y.; Wang, J.; Gu, Z.; Wang, S.; Zhu, W.; Aceña, J. L.; Soloshonok, V. A.; Izawa, K.; Liu, H. Chem. Rev. 2016, 116, 422-518. doi:10.1021/acs.chemrev.5b00392

9. Theodoridis, G. Chapter 4 Fluorine-Containing Agrochemicals: An Overview of Recent Developments. In Advances in Fluorine Science; Tressaud, A., Ed.; Elsevier: Amsterdam, 2006; Vol. 2, pp 121-175. doi:10.1016/S1872-0358(06)02004-5

10. Jeschke, P. Pest Manage. Sci. 2010, 66, 10-27. doi:10.1002/ps.1829

11. Fujiwara, T.; O'Hagan, D. J. Fluorine Chem. 2014, 167, 16-29. doi:10.1016/j.jluchem.2014.06.014

12. Pagliaro, M.; Ciriminna, R. J. Mater. Chem. 2005, 15, 4981-4991. doi:10.1039/b507583c

13. Hird, M. Chem. Soc. Rev. 2007, 36, 2070-2095. doi:10.1039/b610738a

14. Chopra, D.; Row, T. N. G. CrystEngComm 2011, 13, 2175-2186. doi:10.1039/c0ce00538j
15. Berger, R.; Resnati, G.; Metrangolo, P.; Weber, E.; Hulliger, J. Chem. Soc. Rev. 2011, 40, 3496-3508. doi:10.1039/c0cs00221f 16. Hansch, C.; Leo, A.; Taft, R. W. Chem. Rev. 1991, 91, 165-195. doi:10.1021/cr00002a004

17. Leo, A.; Hansch, C.; Elkins, D. Chem. Rev. 1971, 71, 525-616. doi:10.1021/cr60274a001

18. Toulgoat, F.; Alazet, S.; Billard, T. Eur. J. Org. Chem. 2014, 2415-2428. doi:10.1002/ejoc.201301857

19. Xu, X.-H.; Matsuzaki, K.; Shibata, N. Chem. Rev. 2015, 115, 731-764. doi:10.1021/cr500193b

20. Toulgoat, F.; Billard, T. Towards CF3S Group: From Trifluoromethylation of Sulfides to Direct Trifluoromethylthiolation. In Modern Synthesis Processes and Reactivity of Fluorinated Compounds: Progress in Fluorine Science; Groult, H.; Leroux, F.; Tressaud, A., Eds.; Elsevier Science: London, United Kingdom, 2017; pp 141-179. doi:10.1016/B978-0-12-803740-9.00006-8

21. Leroux, F. R.; Manteau, B.; Vors, J.-P.; Pazenok, S. Beilstein J. Org. Chem. 2008, 4, No. 13. doi:10.3762/bjoc.4.13

22. Besset, T.; Jubault, P.; Pannecoucke, X.; Poisson, T. Org. Chem. Front. 2016, 3, 1004-1010. doi:10.1039/C6QO00164E

23. Tlili, A.; Toulgoat, F.; Billard, T. Angew. Chem. 2016, 128 , 11900-11909. doi:10.1002/ange.201603697

24. Romashov, L. V.; Ananikov, V. P. Chem. - Eur. J. 2013, 19 , 17640-17660. doi:10.1002/chem.201302115

25. Wessjohann, L. A.; Schneider, A.; Abbas, M.; Brandt, W. Biol. Chem. 2007, 388, 997-1006. doi:10.1515/BC.2007.138

26. Bodnar, M.; Konieczka, P.; Namiesnik, J. J. Environ. Sci. Health, Part C: Environ. Carcinog. Ecotoxicol. Rev. 2012, 30, 225-252. doi:10.1080/10590501.2012.705164

27. Holben, D. H.; Smith, A. M. J. Am. Diet. Assoc. 1999, 99, 836-843. doi:10.1016/S0002-8223(99)00198-4

28. Brown, K. M.; Arhur, J. R. Public Health Nutr. 2001, 4, 593-599. doi:10.1079/PHN2001143

29. Kyriakopoulos, A.; Behne, D. Rev. Physiol., Biochem. Pharmacol. 2002, 145, 1-46. doi:10.1007/BFb0116430

30. Lu, J.; Holmgren, A. J. Biol. Chem. 2009, 284, 723-727. doi:10.1074/jbc.R800045200

31. Pacuła, A. J.; Kaczor, K. B.; Wojtowicz, A.; Antosiewicz, J.; Janecka, A.; Długosz, A.; Janecki, T.; Ścianowski, J. Bioorg. Med. Chem. 2017, 25, 126-131. doi:10.1016/j.bmc.2016.10.018

32. Combs, G. F., Jr.; Gray, W. P. Pharmacol. Ther. 1998, 79, 179-192. doi:10.1016/S0163-7258(98)00014-X

33. Rayman, M. P. Lancet 2000, 356, 233-241. doi:10.1016/S0140-6736(00)02490-9

34. Abdulah, R.; Miyazaki, K.; Nakazawa, M.; Koyama, H. J. Trace Elem. Med. Biol. 2005, 19, 141-150. doi:10.1016/j.jtemb.2005.09.003

35. Angeli, A.; Tanini, D.; Viglianisi, C.; Panzella, L.; Capperucci, A.; Menichetti, S.; Supuran, C. T. Bioorg. Med. Chem. 2017, 25, 2518-2523. doi:10.1016/j.bmc.2017.03.013

36. Thangamani, S.; Younis, W.; Seleem, M. N. Sci. Rep. 2015, 5 , No. 11596. doi:10.1038/srep11596

37. Singh, N.; Sharpley, A. L.; Emir, U. E.; Masaki, C.; Herzallah, M. M.; Gluck, M. A.; Sharp, T.; Harmer, C. J.; Vasudevan, S. R.; Cowen, P. J.; Churchill, G. C. Neuropsychopharmacology 2016, 41, 1768-1778. doi:10.1038/npp.2015.343

38. Glenadel, Q.; Ismalaj, E.; Billard, T. Eur. J. Org. Chem. 2017, 530-533. doi:10.1002/ejoc.201601526 
39. Zhang, C. J. Chin. Chem. Soc. 2017, 64, 457-463. doi:10.1002/jccs.201600861

40. Chen, C.; Ouyang, L.; Lin, Q.; Liu, Y.; Hou, C.; Yuan, Y.; Weng, Z. Chem. - Eur. J. 2014, 20, 657-661. doi:10.1002/chem.201303934

41. Rong, M.; Huang, R.; You, Y.; Weng, Z. Tetrahedron 2014, 70, 8872-8878. doi:10.1016/j.tet.2014.09.091

42. Zhu, P.; He, X.; Chen, X.; You, Y.; Yuan, Y.; Weng, Z. Tetrahedron 2014, 70, 672-677. doi:10.1016/j.tet.2013.11.093

43. Aufiero, M.; Sperger, T.; Tsang, A. S.-K.; Schoenebeck, F. Angew. Chem. 2015, 127, 10462-10466. doi:10.1002/ange.201503388

44. Hou, C.; Lin, X.; Huang, Y.; Chen, Z.; Weng, Z. Synthesis 2015, 47, 969-975. doi:10.1055/s-0034-1379972

45. Lefebvre, Q.; Pluta, R.; Rueping, M. Chem. Commun. 2015, 51, 4394-4397. doi:10.1039/C4CC10212F

46. Wang, Y.; You, Y.; Weng, Z. Org. Chem. Front. 2015, 2, 574-577. doi:10.1039/C5Q000045A

47. Wu, C.; Huang, Y.; Chen, Z.; Weng, Z. Tetrahedron Lett. 2015, 56, 3838-3841. doi:10.1016/j.tetlet.2015.04.088

48. Fang, W.-Y.; Dong, T.; Han, J.-B.; Zha, G.-F.; Zhang, C.-P. Org. Biomol. Chem. 2016, 14, 11502-11509. doi:10.1039/C6OB02107G

49. Matheis, C.; Krause, T.; Bragoni, V.; Goossen, L. J. Chem. - Eur. J. 2016, 22, 12270-12273. doi:10.1002/chem.201602730

50. Matheis, C.; Wagner, V.; Goossen, L. J. Chem. - Eur. J. 2016, 22, 79-82. doi:10.1002/chem.201503524

51. Tian, Q.; Weng, Z. Chin. J. Chem. 2016, 34, 505-510. doi:10.1002/cjoc.201600052

52. Wang, J.; Zhang, M.; Weng, Z. J. Fluorine Chem. 2017, 193, 24-32. doi:10.1016/j.jfluchem.2016.11.006

53. Dürr, A. B.; Fisher, H. C.; Kalvet, I.; Truong, K.-N.; Schoenebeck, F. Angew. Chem., Int. Ed. 2017, 56, 13431-13435. doi:10.1002/anie.201706423

54. Dale, J. W.; Emeléus, H. J.; Haszeldine, R. N. J. Chem. Soc. 1958, 2939-2945. doi:10.1039/JR9580002939

55. Yarovenko, N. N.; Shemanina, V. N.; Gazieva, G. B. Russ. J. Gen. Chem. 1959, 29, 924-927.

56. Yagupol'skii, L. M.; Voloshchuk, V. G. Russ. J. Gen. Chem. 1966, 36, 173-174.

57. Voloshchuk, V. G.; Yagupol'skii, L. M.; Syrova, G. P.; Bystrov, V. P. Russ. J. Gen. Chem. 1967, 37, 105-108.

58. Yagupol'skii, L. M.; Voloshchuk, V. G. Russ. J. Gen. Chem. 1967, 37, 1463-1465.

59. Yagupol'skii, L. M.; Voloshchuk, V. G. Russ. J. Gen. Chem. 1968, 38, 2426-2429.

60. Marsden, C. J. J. Fluorine Chem. 1975, 5, 401-422. doi:10.1016/S0022-1139(00)82499-9

61. Haas, A.; Praas, H.-W. Chem. Ber. 1992, 125, 571-579. doi:10.1002/cber.19921250308

62. Magnier, E.; Vit, E.; Wakselman, C. Synlett 2001, 1260-1262. doi:10.1055/s-2001-16050

63. Magnier, E.; Wakselman, C. Collect. Czech. Chem. Commun. 2002, 67, 1262-1266. doi:10.1135/cccc20021262

64. Glenadel, Q.; Ismalaj, E.; Billard, T. J. Org. Chem. 2016, 81, 8268-8275. doi:10.1021/acs.joc.6b01344

65. Ghiazza, C.; Glenadel, Q.; Tlili, A.; Billard, T. Eur. J. Org. Chem. 2017, 3812-3814. doi:10.1002/ejoc.201700643

66. Ghiazza, C.; Tlili, A.; Billard, T. Molecules 2017, 22, 833-841. doi:10.3390/molecules22050833

67. Ghiazza, C.; Billard, T.; Tlili, A. Chem. - Eur. J. 2017, 23, 10013-10016. doi:10.1002/chem.201702028
68. Glenadel, Q.; Ghiazza, C.; Tlili, A.; Billard, T. Adv. Synth. Catal. 2017, 359, 3414-3420. doi:10.1002/adsc.201700904

\section{License and Terms}

This is an Open Access article under the terms of the Creative Commons Attribution License

(http://creativecommons.org/licenses/by/4.0), which permits unrestricted use, distribution, and reproduction in any medium, provided the original work is properly cited.

The license is subject to the Beilstein Journal of Organic Chemistry terms and conditions:

(http://www.beilstein-journals.org/bjoc)

The definitive version of this article is the electronic one which can be found at:

doi: $10.3762 /$ bjoc. 13.260 